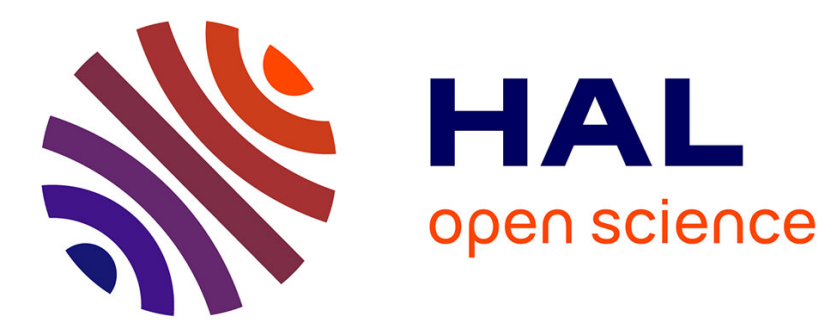

\title{
Random Matrix Theory Based Resource Allocation in Correlated MIMO Systems with ARQ Feedback
}

Marie Zwingelstein, Mérouane Debbah

\section{To cite this version:}

Marie Zwingelstein, Mérouane Debbah. Random Matrix Theory Based Resource Allocation in Correlated MIMO Systems with ARQ Feedback. IEEE Communications Letters, 2014, 18 (5), pp.793-796. 10.1109/LCOMM.2014.031414.140214 . hal-01098948

\section{HAL Id: hal-01098948 \\ https://hal.science/hal-01098948}

Submitted on 17 Jan 2015

HAL is a multi-disciplinary open access archive for the deposit and dissemination of scientific research documents, whether they are published or not. The documents may come from teaching and research institutions in France or abroad, or from public or private research centers.
L'archive ouverte pluridisciplinaire HAL, est destinée au dépôt et à la diffusion de documents scientifiques de niveau recherche, publiés ou non, émanant des établissements d'enseignement et de recherche français ou étrangers, des laboratoires publics ou privés. 


\title{
Random Matrix Theory Based Resource Allocation in Correlated MIMO Systems with ARQ Feedback
}

\author{
Marie Zwingelstein-Colin and Mérouane Debbah, Senior Member, IEEE
}

\begin{abstract}
We consider resource allocation under partial feedback in a spatially correlated MIMO link, when the ARQ protocol is implemented at the MAC layer. We propose a design framework, which makes use of results from random matrix theory (RMT), to find the rate as well as the input covariance matrix that maximize the long term goodput. We consider partial feedback in terms of positive/negative acknowledgment bits (ACK/NAK), which comes essentially for free since they are always present in the signaling of the upper layers. We provide explicit expressions of the long term goodput, which, in association with a RMT based approximation of the mutual information enable us to optimize the resource allocation problem. Interestingly, the simulations show that the asymptotic optimization analysis is still valid for MIMO sizes as small as $2 \times 2$.
\end{abstract}

Index Terms-MIMO, resource allocation, random matrix theory, ARQ, partial feedback.

\section{BACKGROUND AND MOTIVATION}

$\mathbf{R}$ ESOURCE allocation is a widely studied subject (e.g. [1] and references therein) for which the assumptions may be of two kinds: perfect channel state information at the transmitter (CSI-T), and partial CSI-T, also known as partial feedback. Only the latest is acceptable from a practical point of view as soon as the number of degrees of freedom in the system gets large (MIMO, wideband or/and multi-user systems for example).

The objective of this paper is to present a design framework to optimize the long term goodput by using the ACK/NAK bits provided by ARQ. This can be viewed as a cross-layer design in the sense that physical layer parameters are optimized based on (ARQ) information provided at the MAC layer [2]. We apply results from Random Matrix Theory (RMT) to estimate the average received SNR and determine the rate and the input covariance matrix to maximize the goodput. To the best of the authors' knowledge, RMT results have never been applied to the resource allocation problem for MIMO-ARQ systems. Importantly, general considerations about the optimal transmission scheme without perfect CSI-T (training-based methods, blind methods, error-rate based methods...) are out of the scope of this paper. The proposed framework can be seen as a proof of concept that acknowledgment bits can be used for the resource allocation problem in a MIMO-ARQ setup, although it is probable that optimal solutions would be, e.g., mixed training-ARQ based.

Manuscript received January 29, 2014. The associate editor coordinating the review of this letter and approving it for publication was S. Jin.

M. Zwingelstein-Colin is with the University of Valenciennes, France (email: marie.zwingelstein-colin@univ-valenciennes.fr).

Mérouane Debbah is with the SUPELEC - Alcatel-Lucent Chair on Flexible Radio, Gif sur Yvette, France.

Digital Object Identifier 10.1109/LCOMM.2014.031414.140214
Rate adaptation based on partial CSI-T for point to point SISO channels with HARQ has been investigated in [3]. It was shown that HARQ can provide an important rate advantage as compared to a non HARQ scheme when there is not enough channel selectivity. Rate adaptation for goodput optimization is also considered in [4], [5] for SISO and MIMO links respectively. Finally, in [6], a probe intervall is developped to perform rate-adaptation in wireless SISO links with ARQ feedback. Also, in contrast with [5], the work presented in this paper provides the following three original contributions: 1) Spatial correlation is taken into account. 2) Results from RMT are exploited to derive analytical formulations. 3) SNR is estimated based on the ARQ feedback bits.

This paper is organized as follows. After the problem formulation in section II, in section III we provide and evaluate an approximated analytical formulation of the long term goodput, based on RMT. We then present and evaluate our framework for resource allocation in section IV and V respectively, and conclude in section VI. Notations: Bold letters denote vector or matrix quantities, superscript ${ }^{H}$ the hermitian transpose and superscript * the optimum.

\section{Problem Formulation}

We consider a point to point $n_{t} \times n_{r}$ correlated MIMO link, under the frequency-flat block-fading scenario. At block number $k$, the $\ell$-th received data symbol is

$$
\mathbf{y}_{\ell}=\sqrt{\frac{\rho}{n_{t}}} \mathbf{H}_{k} \mathbf{x}_{\ell}+\mathbf{n}_{\ell}
$$

where $\mathbf{x}_{\ell} \in \mathcal{C}^{n_{t}}$ is the input vector, $\mathbf{H}_{k} \in \mathcal{C}^{n_{r} \times n_{t}}$ is the channel matrix, $\mathbf{n}_{\ell} \in \mathcal{C}^{n_{r}}$ is the unit variance white Gaussian noise vector and the received SNR $\rho$ is assumed to be constant over $T \gg 1$ blocks. Spatial correlation follows the Kronecker model, that is $\mathbf{H}_{k}=\mathbf{R}_{r}^{\frac{1}{2}} \boldsymbol{\Theta}_{k} \mathbf{R}_{t}^{\frac{1}{2}}$, the elements of $\boldsymbol{\Theta}_{k}$ being i.i.d. $\sim \mathcal{C N}(0,1), \operatorname{Tr}\left\{\mathbf{R}_{t}\right\}=n_{t}$ and $\operatorname{Tr}\left\{\mathbf{R}_{r}\right\}=n_{r}$. For a uniform antenna array, classical correlation models are parameterized by the distance $d$ between two adjacent antennas, reflecting the fact that they depend almost entirely on the geometrical structure of the antenna arrays. ${ }^{1}$ At the MAC layer, the ARQ protocol feedbacks a one-bit ACK in case of successful packet reception and a one-bit NAK otherwise. One ARQ packet lasts one block of data (slow-fading scenario), and we assume perfect error detection at the receiver. We also consider strong channel coding so that the source of

\footnotetext{
${ }^{1}$ In the sequel, we use the spatial correlation model presented in [7] in which the the correlation matrices are Toeplitz matrices which are based on the vector $\left(1, e^{-\frac{d}{\lambda}}, \ldots, e^{-(n-1) \frac{d}{\lambda}}\right)$, where $\lambda$ is the wavelength.
} 


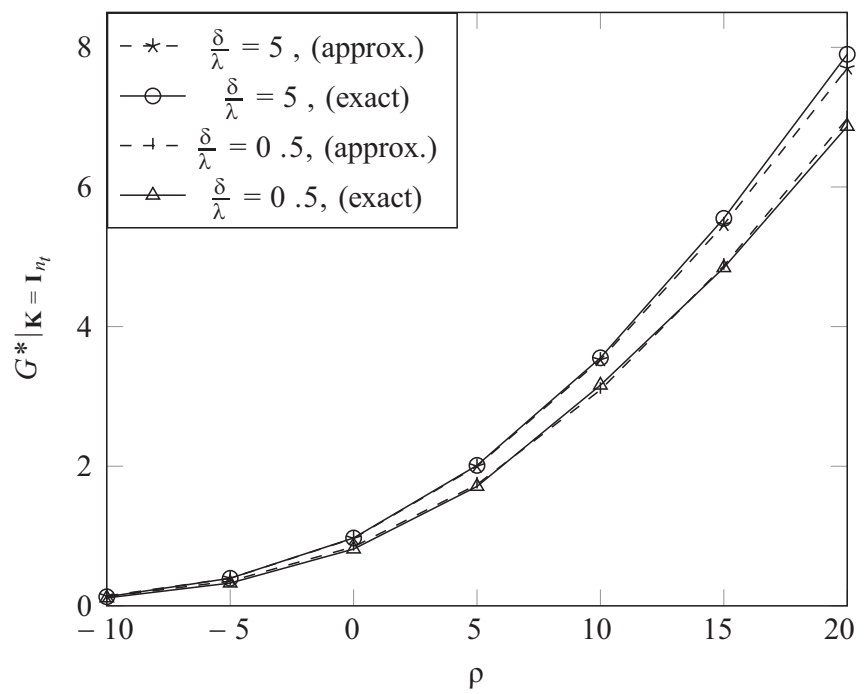

Fig. 1. Optimal goodput of a 2x2 MIMO system from Monte-Carlo simulation (exact) and Gaussian approximation (app.), with high $\left(\frac{d}{\lambda}=0.5\right)$ and low $\left(\frac{d}{\lambda}=5\right)$ spatial correlation.

transmission errors is limited to the outage of the mutual information. As a result, the packet error rate is

$$
\epsilon(\rho, R, \mathbf{K})=\operatorname{Pr}\left[\log _{2} \operatorname{det}\left(\mathbf{I}_{n_{r}}+\frac{\rho}{n_{t}} \mathbf{H}_{k} \mathbf{K H}_{k}^{H}\right)<R\right]
$$

where the transmission rate, $R$, and the covariance of the input, $\mathbf{K}=\mathbb{E}\left\{\mathbf{x}_{\ell} \mathbf{x}_{\ell}^{H}\right\}$, are kept constant within a block. Since, in accordance with the block-fading scenario, successive packetoutages are independent, the long term goodput is :

$$
G=\frac{R}{\mathbb{E}\{X\}}=R \frac{1-\epsilon(\rho, R, \mathbf{K})}{1-\epsilon(\rho, R, \mathbf{K})^{M}}
$$

where $X$ is the geometrically distibuted discrete random variable representing the number of transmission attempts of each packet and $M$ is the maximum number of ARQ retransmissions (which we consider to be $\infty$ in the sequel, for simplicity of the analysis). For a given $\rho, \mathbf{R}_{t}$ and $\mathbf{R}_{r}$, the goodput $G$ is a function of the transmission rate $R$ and the input covariance matrix $\mathbf{K}$. In this paper, we design a framework for resource allocation, in which the transmitter exploits the ARQ feedback bits to estimate $\rho$, and determinates $R$ and $\mathbf{K}$ to maximize $G$ based on the estimated $\hat{\rho}$ of $\rho$.

\section{RANDOM MATRIX THEORY BASED GAUSSIAN APPROXIMATION}

Here, we show that the first and second order asymptotic approximations of the mutual information of large dimensional multi-antenna channels provided in [8] can be used as a tool for our resource allocation problem, under both high and low spatial correlation, even if the number of antennas at the transmitter and at the receiver is kept as low as 2. First, we recall these approximations, which are given for $\mathbf{K}=\mathbf{I}_{n_{t}}$ (generalization to $\mathbf{K} \neq \mathbf{I}_{n_{t}}$ is examined in section IV). Then, we examine how the optimized goodput is impacted by these approximations.

First order approximation of the mutual information of large MIMO channels (from [8], theorem 1):

$$
\mathbb{E}\left\{\log _{2} \operatorname{det}\left(\mathbf{I}_{n_{r}}+\frac{\rho}{n_{t}} \mathbf{H}_{k} \mathbf{H}_{k}^{H}\right)\right\}=\mu_{I}(\rho, \mathbf{D}, \tilde{\mathbf{D}})+\mathcal{O}\left(\frac{1}{n_{t}}\right) \text { as }
$$
$n_{t}, n_{r} \rightarrow \infty^{2}$, where $\mathbf{D}$ and $\tilde{\mathbf{D}}$ are the diagonal matrices of eigenvalues of $\mathbf{R}_{r}$ and $\mathbf{R}_{t}$ respectively, and

$$
\begin{aligned}
\mu_{I}(\rho, \mathbf{D}, \tilde{\mathbf{D}})= & \log _{2} \operatorname{det}\left(\mathbf{I}_{n_{t}}+\rho \delta \tilde{\mathbf{D}}\right)+ \\
& \log _{2} \operatorname{det}\left(\mathbf{I}_{n_{r}}+\rho \tilde{\delta} \mathbf{D}\right)-n_{t} \rho \delta \tilde{\delta} \log _{2}(e)
\end{aligned}
$$

where $(\delta, \tilde{\delta})$ is the unique positive solution of the system

$$
\left\{\begin{array}{l}
\delta=\frac{1}{n_{t}} \operatorname{tr} \mathbf{D}\left(\mathbf{I}_{n_{r}}+\rho \tilde{\delta} \mathbf{D}\right)^{-1} \\
\tilde{\delta}=\frac{1}{n_{t}} \operatorname{tr} \tilde{\mathbf{D}}\left(\mathbf{I}_{n_{t}}+\rho \delta \tilde{\mathbf{D}}\right)^{-1}
\end{array}\right.
$$

Second order approximation of the mutual information of large MIMO channels (from [8], theorem 2):

$$
\frac{\log _{2} \operatorname{det}\left(\mathbf{I}_{n_{r}}+\frac{\rho}{n_{t}} \mathbf{H}_{k} \mathbf{H}_{k}^{H}\right)-\mu_{I}(\rho, \mathbf{D}, \tilde{\mathbf{D}})}{\sigma_{I}(\rho, \mathbf{D}, \tilde{\mathbf{D}})} \text { converges in dis- }
$$

tribution to $\mathcal{N}(0,1)$ as $n_{t}, n_{r} \rightarrow \infty^{2}$, where

$$
\sigma_{I}^{2}(\rho, \mathbf{D}, \tilde{\mathbf{D}})=-\log _{2}\left(1-\rho^{2} \gamma \tilde{\gamma}\right)
$$

and

$$
\begin{aligned}
& \gamma=\frac{1}{n_{t}} \operatorname{tr}^{2}\left(\mathbf{I}_{n_{t}}+\rho \tilde{\delta} \mathbf{D}\right)^{-2} \\
& \tilde{\gamma}=\frac{1}{n_{t}} \operatorname{tr} \tilde{\mathbf{D}}^{2}\left(\mathbf{I}_{n_{t}}+\rho \delta \tilde{\mathbf{D}}\right)^{-2}
\end{aligned}
$$

Therefore, for $\mathbf{K}=\mathbf{I}_{n_{t}}$, one can write

$$
G(\rho, R, \mathbf{K}) \approx R\left(1-Q\left(\frac{\mu_{I}(\rho, \mathbf{D}, \tilde{\mathbf{D}})-R}{\sigma_{I}(\rho, \mathbf{D}, \tilde{\mathbf{D}})}\right)\right)
$$

We now compare the rate optimized goodput $\left.G^{*}\right|_{\mathbf{K}=\mathbf{I}_{n_{t}}}=$ $\max _{R} R\left(1-\epsilon\left(\rho, R, \mathbf{I}_{n_{t}}\right)\right)$ obtained by Monte-Carlo simulation (exact) to that given by the approximation (5). Fig. 1 presents $\left.G^{*}\right|_{\mathbf{K}=\mathbf{I}_{n_{t}}}$ vs $\rho$ for $n_{t}=n_{r}=2, \rho$ varying from $-10 \mathrm{~dB}$ to $25 \mathrm{~dB}$ and $\frac{d}{\lambda} \in\{0.5,5\}$ (thus covering both high and low spatial correlation [7] senarios). We can see a very good match between the exact and approximated curves, even if the number of antennas is as low as $2 \times 2$.

\section{Resource Allocation Framework}

Resource allocation is performed over two phases, without using any training sequence. Hence, useful data is transmitted over both phases. $R$ and $\mathbf{K}$ are derived in such a way that $\rho$ is estimated in phase I and the goodput is maximized in phase II. Both phases exploit RMT-based approximations (5). We begin our discussion with phase II.

Phase II $\left(T_{p}+1 \leq k \leq T\right)$ : Noting that the optimal value of $\mathbf{K}$ in terms of outage is not known in general, the problem of determining the one that maximizes the goodput seems prohibitive. We concentrate on a suboptimal solution that consists in 1) adopting the ergodic capacity achieving covariance matrix structure, which is known to correspond to $\mathbf{K}=\mathbf{U}_{t} \boldsymbol{\Sigma} \mathbf{U}_{t}^{H}$, where $\mathbf{U}_{t}$ is the eigenvector matrix of $\mathbf{R}_{t}$, see

\footnotetext{
${ }^{2}$ but the ratio tends to a constant.That is $n_{r}\left(n_{t}\right)$ is a sequence of integers such that $0<\liminf _{n_{t} \rightarrow \infty} \frac{n_{r}\left(n_{t}\right)}{n_{t}} \leq \limsup _{n_{t} \rightarrow \infty} \frac{n_{r}\left(n_{t}\right)}{n_{t}}<\infty$.
} 
references [9], [10], and 2) optimizing the goodput ${ }^{3}$ through power allocation over the eigenvalues $\zeta(i), i=1, \ldots, n_{t}$ $(\boldsymbol{\Sigma}=\operatorname{diag}(\zeta(i)))$ and optimization of the rate $R$. Note that a similar approach was used in [11] where the form of the covariance matrix that achieves the capacity in the Rayleigh fading case was used to investigate the capacity under Rician distribution. Noting that $\log _{2} \operatorname{det}\left(\mathbf{I}_{n_{r}}+\frac{\hat{\rho}}{n_{t}} \mathbf{H}_{k} \mathbf{K H}_{k}^{H}\right)=$ $\log _{2} \operatorname{det}\left(\mathbf{I}_{n_{r}}+\frac{\hat{\rho}}{n_{t}} \mathbf{H}_{k}^{\prime} \mathbf{H}_{k}^{\prime} H\right)$, where $\mathbf{H}_{k}^{\prime}=\mathbf{D}^{\frac{1}{2}} \mathbf{\Theta}^{\frac{1}{2}} \boldsymbol{\Sigma}^{\frac{1}{2}}$, this goodput optimization problem can be formulated as the problem 1 below:

Problem 1 (Goodput optimization):

$$
\begin{aligned}
G^{*}(\hat{\rho})=\max _{R, \boldsymbol{\Sigma}} & R\left(1-Q\left(\frac{\mu_{I}(\hat{\rho}, \mathbf{D}, \tilde{\mathbf{D}} \boldsymbol{\Sigma})-R}{\sigma_{I}(\hat{\rho}, \mathbf{D}, \tilde{\mathbf{D}} \boldsymbol{\Sigma})}\right)\right) \\
\text { subject to } \quad & R \geq 0, \\
& \operatorname{Tr}(\boldsymbol{\Sigma}) \leq n_{t} .
\end{aligned}
$$

We solved it numerically using active-set nonlinear programming tools.

Phase I $\left(1 \leq k \leq T_{p}\right)$ : Unlike estimation techniques based on training sequences (that do not contribute in useful data transmission), the packets not in outage during phase I do contribute to the goodput. We modify the algorithm developed in [6] in the context of SISO transmission of uncoded QAM (shown to converge to an efficient and consistent estimator) whose general principle, adapted to our context, is: iteration $k$ corresponds to the transmission of one packet, at a rate $R_{k}$, and with input covariance $\mathbf{K}_{k}=\mathbf{U}_{t} \boldsymbol{\Sigma}_{k} \mathbf{U}_{t}^{H}$. At each iteration, $\hat{\rho}$ is updated based on the ARQ bit (denoted $F_{k}$, equal to 1 in case of NAK and to 0 otherwise) that is fed back in relation to that packet, and so are the rate and the input covariance matrix. The updating equations are derived so that the CramérRao lower bound is achieved by the estimator ${ }^{4}$ [12]. In more details: 1 . At block index $k=1$, choose arbitrarily a rate $R_{1}$ and an SNR estimate $\hat{\rho}_{1}$, and compute $\boldsymbol{\Sigma}_{1}$ according to

$$
\begin{array}{cl}
\boldsymbol{\Sigma}_{1}=\arg \max _{\boldsymbol{\Sigma}} & R_{1}\left(1-Q\left(\frac{\mu_{I}\left(\hat{\rho}_{1}, \mathbf{D}, \tilde{\mathbf{D}} \boldsymbol{\Sigma}\right)-R_{1}}{\sigma_{I}\left(\hat{\rho}_{1}, \mathbf{D}, \tilde{\mathbf{D}} \boldsymbol{\Sigma}\right)}\right)\right) \\
\text { s.t. } & \operatorname{Tr}(\boldsymbol{\Sigma}) \leq n_{t} .
\end{array}
$$

Transmit packet 1 at a rate $R_{1}$, with input covariance $\Sigma_{1}$, and read the feed-backed bit $F_{1}$.

2. For block indexes $2 \leq k \leq T_{p}$ :

a) Update

$$
\hat{\rho}_{k}=\hat{\rho}_{k-1}+\frac{F_{k-1}-\epsilon\left(\hat{\rho}_{k-1}, R_{k-1}, \mathbf{U}_{t} \boldsymbol{\Sigma}_{k-1} \mathbf{U}_{t}^{H}\right)}{(k-1)^{\beta} \times \epsilon^{\prime}\left(\hat{\rho}_{k-1}, R_{k-1}, \mathbf{U}_{t} \mathbf{\Sigma}_{k-1} \mathbf{U}_{t}^{H}\right)}
$$

where $\epsilon^{\prime}\left(\rho, R, \mathbf{U}_{t} \boldsymbol{\Sigma} \mathbf{U}_{t}^{H}\right) \stackrel{\text { def }}{=} \frac{\partial}{\partial \rho} \epsilon\left(\rho, R, \mathbf{U}_{t} \boldsymbol{\Sigma} \mathbf{U}_{t}^{H}\right) \cdot \beta \epsilon$ $(0,1]$ controls the tradeoff between the speed of convergence and the variance of the estimation error.

\footnotetext{
${ }^{3}$ Since $\mathbf{R}_{t}$ and $\mathbf{R}_{r}$ depend almost entirely on the geometry of the antenna array, they are very little sensitive to receiver mobility, and can be assumed to be (almost) constant over $\gg T$ blocks. Therefore, we consider that they are known at the transmitter without any penalty in the rate.

${ }^{4}$ We denote by $\Phi(\rho, R, \boldsymbol{\Sigma})$ the Fisher information relative to $F_{k}$ $\left(\Phi(\rho, R, \boldsymbol{\Sigma}) \stackrel{\text { def }}{=} \underset{f_{k}}{\mathbb{E}}\left\{\frac{\partial}{\partial \rho} \log \operatorname{Pr}\left[F_{k}=f_{k} \mid \rho, R, \boldsymbol{\Sigma}\right]\right\}\right)$.
}

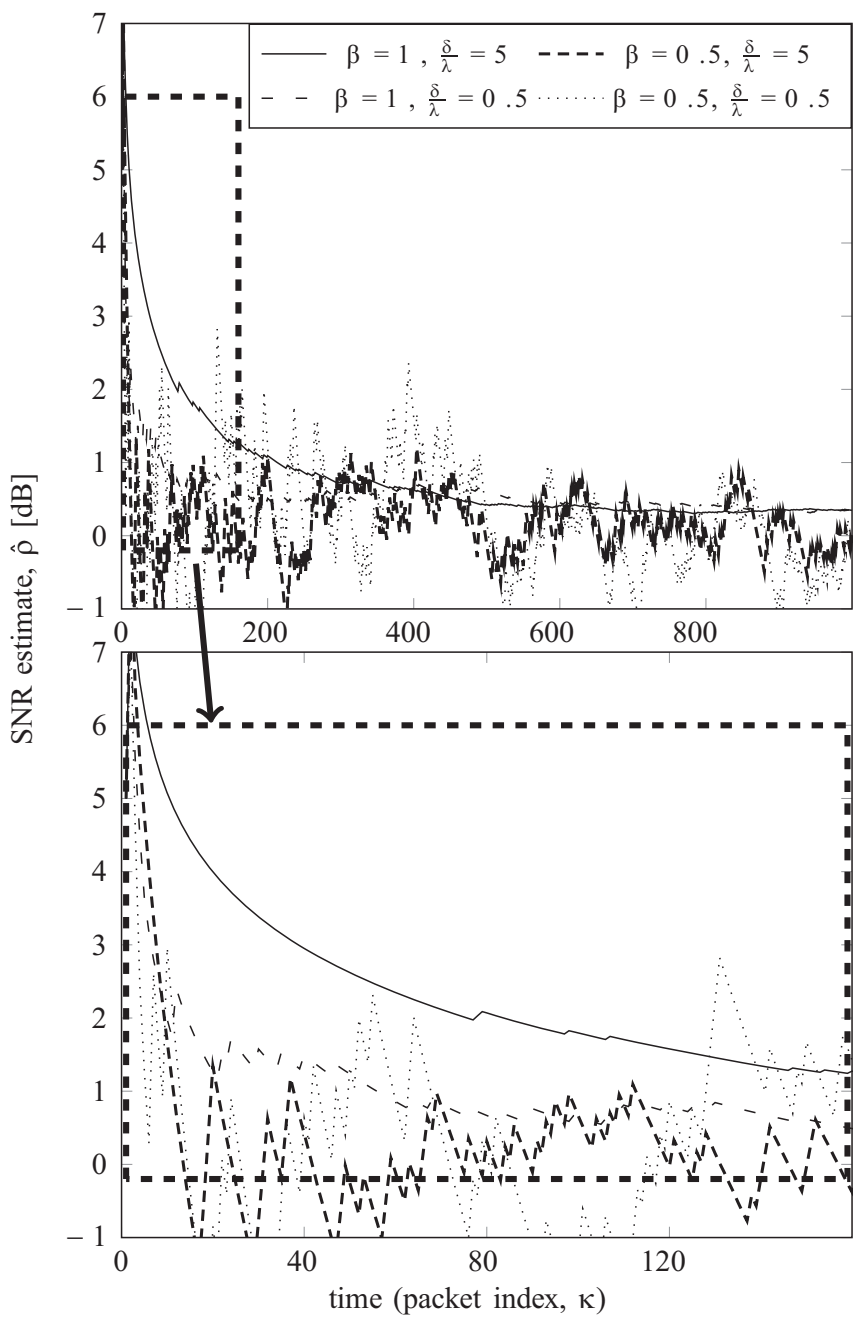

Fig. 2. Illustration of the trajectory of the SNR estimator. $\beta \in\{0.5,1\}$ and $\frac{d}{\lambda} \in\{0.5,5\}$.

b) Choose $R_{k}$ and $\boldsymbol{\Sigma}_{k}$ as

$$
\begin{gathered}
\left(R_{k}, \boldsymbol{\Sigma}_{k}\right)=\arg \max _{R, \boldsymbol{\Sigma}} \Phi\left(\hat{\rho}_{k}, R, \boldsymbol{\Sigma}\right) \\
\text { s.t. } R \geq 0, \operatorname{Tr}(\boldsymbol{\Sigma}) \leq n_{t}
\end{gathered}
$$

c) Transmit packet $k$ at a rate $R_{k}$, with input covariance $\boldsymbol{\Sigma}_{k}$, and read the feed-backed bit $F_{k}$.

The optimized goodput, averaged over both phases, can be written:

$$
G_{\mathrm{av}}^{*}=\frac{1}{T} \sum_{k=1}^{T_{p}} R_{k}\left(1-F_{k}\right)+\frac{T-T_{p}}{T} \times G^{*}(\hat{\rho})
$$

where $\frac{1}{T} \sum_{k=1}^{T_{p}} R_{k}\left(1-F_{k}\right)$ is the contribution of phase I to the goodput and $G^{*}(\hat{\rho})$ is the result of problem 1 .

\section{Performance Results}

We first evaluate the performance of the goodput optimization scheme of phase II against uniform power allocation (PA) and beamforming (BF) ${ }^{5}$, assuming $\hat{\rho}=\rho$. The evolution of the goodput with $\frac{d}{\lambda}$ is represented on Fig. 2. The curves

\footnotetext{
${ }^{5}$ uniform: $\mathbf{K}=\mathbf{I}_{n_{t}}$; BF: all the power is allocated to the maximum eigenvalue of $\mathbf{R}_{t}$.
} 


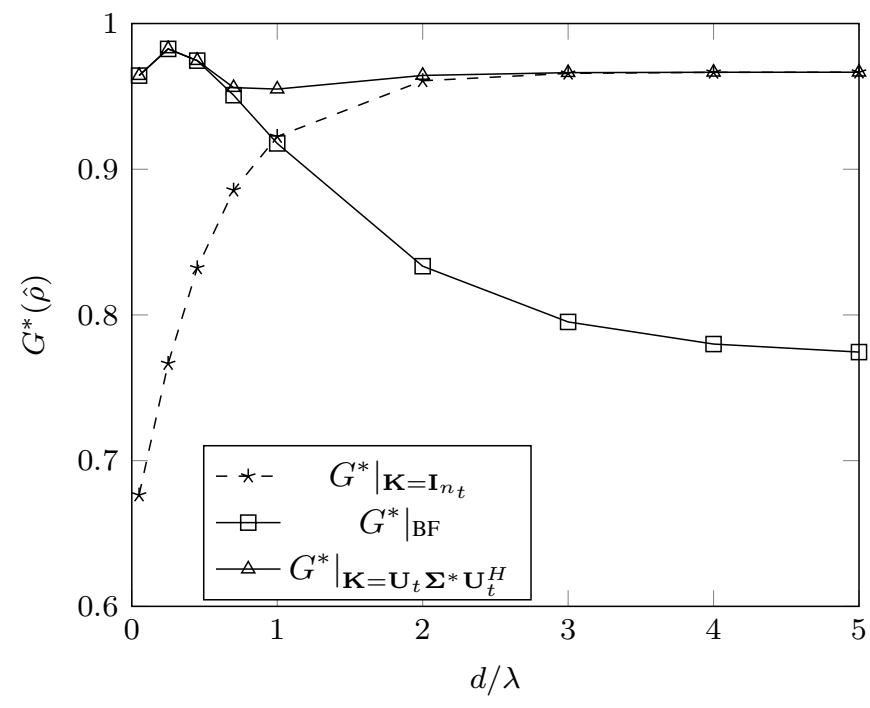

Fig. 3. Optimized goodput (phase II only) against uniform PA and BF. $\hat{\rho}=0$ $\mathrm{dB}, n_{t}=n_{r}=2$.

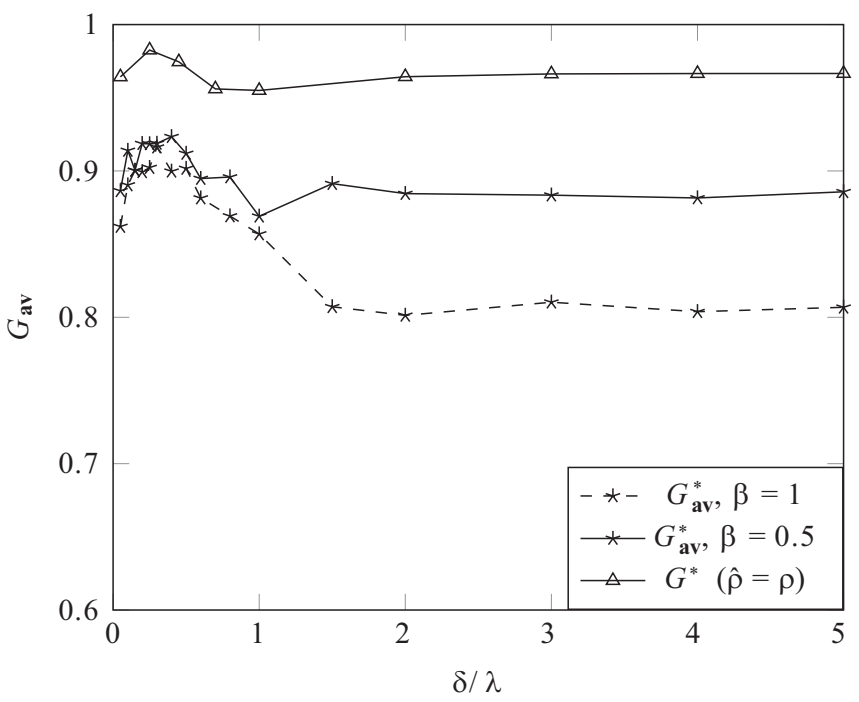

Fig. 4. Optimized goodput, averaged over both phases. $n_{t}=n_{r}=2, \rho=0$ $\mathrm{dB}, \rho_{1}=5 \mathrm{~dB}, T_{p}=100, T=1000$.

confirm the intuition that $\mathbf{K}^{*} \rightarrow \mathbf{I}_{n_{t}}$ in low correlated environments, and $\mathbf{K}^{*} \rightarrow \mathrm{BF}$ in highly correlated environments. For intermediate correlation, our proposal $\mathbf{K}^{*}$ outperforms both $\mathbf{K}=\mathbf{I}_{n_{t}}$ and $\mathrm{BF}$.

The trajectory of the SNR estimator in phase I is illustrated in Fig. 3. We arbitraritly fixed $R_{1}=1 \mathrm{bps} / \mathrm{Hz}$ and $\hat{\rho}_{1}=5 \mathrm{~dB}$ $(\rho=0 \mathrm{~dB})$. For $\beta=0.5$, we can see that it takes less time to go to the steady state than for $\beta=1$, but the estimator variance is higher. Also, the rate of convergence is higher in the presence of high spatial correlation $\left(\frac{d}{\lambda}=0.5\right)$ when $\beta=1$.

In order to investigate the impact of the SNR estimation on the goodput, Fig. 4 represents $G_{\mathrm{av}}^{*}$ as a function of $\frac{d}{\lambda}$ for $\beta \in\{0.5,1\}, T_{p}=100$ and $T=1000$, as well as $G^{*}(\rho)$ for comparison purpose. We observe that $G_{\mathrm{av}}^{*}$ reaches around $80-90 \%$ of $G_{\mathrm{av}}^{*}$ depending on the value of $\beta$ (which could be improved by optimizing beta).

\section{CONCLUSION}

In this paper we designed a framework for rate and input covariance matrix optimization, when only ARQ feedback is present, in a spatially correlated environment. We exploited results from RMT to provide a convenient formulation of the goodput which was used in the optimization process. Extending the work provided in [6], we proposed a two phases allocation framework, both contributing to useful data transmission, where the SNR is inferred from binary ACK/NAK feedback and used as an input to the goodput optimization problem. Possible extension of this work is to consider mixed trainingARQ based solutions for the resource allocation problem.

\section{REFERENCES}

[1] I. Wong and B. Evans, Resource Allocation in Multiuser Multicarrier Wireless Systems. Springer, 2008.

[2] X. Lin, N. B. Shroff, and R. Srikant, "A tutorial on cross-layer optimization in wireless networks," IEEE J. Sel. Areas Commun., vol. 24, pp. 1452-1463, Aug. 2006.

[3] P. Wu and N. Jindal, "Performance of Hybrid-ARQ in block-fading channels: a fixed outage probability analysis," IEEE Trans. Commun., vol. 58, pp. 1129-1141, Apr. 2010.

[4] — , "Coding versus ARQ in fading channels: how reliable should the PHY be?" in Proc. 2009 IEEE Globecom, pp. 1-6.

[5] M. Zwingelstein-Colin and M. Debbah, "Physical layer reliability vs ARQ in MIMO block-fading channels," in Proc. 2012 IEEE WCNC, pp. 1670-1674.

[6] C. E. Koksal and P. Schniter, "Robust rate-adaptative wireless communication using ACK/NAK-feedback," IEEE Trans. Signal Process., vol. 60, pp. 1752-1765, Apr. 2012.

[7] C. Oestges, M. Guillaud, and M. Debbah, "Multi-polarized MIMO communications: channel model, mutual information and array optimization," in Proc. 2007 IEEE WCNC, pp. 1057-1061.

[8] W. Hachem, O. Khorunzhiy, P. Loubaton, J. Najim, and L. Pastur, "A new approach for mutual information analysis of large dimensional multiantenna channels," IEEE Trans. Inf. Theory, vol. 54, pp. 3987-4004, Sep. 2008.

[9] E. Jorswieck and H. Boche, "Optimal transmission strategies and impact of correlation in multi-antenna systems with different types of channel state information," IEEE Trans. Signal Process., vol. 52, pp. 3440-3453, Dec. 2004.

[10] A. M. Tulino, A. Lozano, and S. Verdu, "Impact of antenna correlation on the capacity of multiantenna channels," IEEE Trans. Inf. Theory, vol. 51, pp. 2491-2509, Jul. 2005.

[11] S. K. Jayaweera and V. Vincent, "On the capacity of multi-antenna systems in the presence of Rician fading," in Proc. 2002 IEEE VTC Spring, vol. 4, pp. 1963-1967.

[12] M. B. Nevelson and R. Z. Hasminskii, Stochastic Approximation and Recursive Estimation. American Mathematical Society, 1976. 